\title{
Resurfacing of Two Separate Digital Defects Using a Single Fascial Free Flap with Neosyndactylization
}

\author{
Fernando A. Herrera Jr., MD ${ }^{1,2}$ Karen M. Horton, MD ${ }^{1}$ Ryan Brennan, BS ${ }^{2}$ Gregory M. Buncke, MD ${ }^{1}$ \\ Rudolph F. Buntic, $M D^{1}$ \\ ${ }^{1}$ The Buncke Clinic and Division of Microsurgery, California Pacific \\ Medical Center, San Francisco, California \\ 2 Division of Plastic and Hand Surgery, Medical University of South \\ Carolina, Charleston, South Carolina \\ Address for correspondence Fernando A. Herrera Jr, MD, Division of \\ Plastic and Hand Surgery, Department of Surgery, Medical University \\ of South Carolina, Charleston, SC 29425 \\ (e-mail: fherrera00@yahoo.com).
}

J Reconstr Microsurg Open 2018;3:e87-e90.

\begin{abstract}
We report a case of a 20-year-old patient who sustained a mutilating crush injury to the left-hand index and ring finger volar surface measuring $5 \times 6 \mathrm{~cm}$ from the distal end of the proximal phalanx to the fingertips. After thorough debridement and stabilization of the skeletal injury, a radial forearm fascia only free flap measuring $6 \times 7 \mathrm{~cm}$ was used to resurface the distal soft tissue volar defect of two adjacent fingers. Digital nerve grafting was also required, and this was done using autologous nerve graft from the lateral antebrachial cutaneous nerve. This thin fascia only flap allows for stable soft tissue coverage and provides a gliding surface for the underlying tendons. The neosyndactylized digits were safely divided at 3-month follow-up, and excellent

Keywords

- free fascia flap

- neosyndactyly

- digital resurfacing functional and aesthetic results were achieved. The radial forearm fascia is a thin, durable, and pliable tissue that is based on the radial artery as a vascular pedicle. We consider this free fascial flap as a valuable option for coverage of multiple complex distal digit injuries using a single flap and highly recommend its use.
\end{abstract}

Traumatic hand and digit injuries resulting in soft tissue loss pose many challenges for the reconstructive surgeon. These injuries may result in composite tissue loss to digits and require soft tissue coverage as well as bone, tendon, and nerve grafts. Coverage of exposed structures, such as tendons, bones, vessels, or nerves at the dorsal and palmer surfaces of the hand, requires thin, supple tissue to provide adequate range of motion and a satisfying aesthetic result. The use of local and regional flaps when possible to treat these injuries is a popular choice. ${ }^{1-4}$ However, due to the limited amount of expendable tissue in the hand and fingers, this may result in a functional limitation at the donor site or simply may not be possible due to the surrounding zone of injury (multiple digital crush or avulsion injuries). Advancements in microsurgical techniques have allowed the use of free tissue transfers to treat these defects in the distal hand and fingers, allowing for improved range of motion and a more aesthetically pleasing appearance of the traumatized upper extremity using thin pliable flaps. ${ }^{5-7}$ We discuss a case of simultaneous coverage of two large digital defects with a single thin fascial free flap with neosyndactylization.

\section{Case Report}

A 20-year-old, right hand dominant male sustained a significant crush injury to his left hand after a motor vehicle rollover collision. Initial evaluation of his injuries revealed a complex soft tissue avulsion injury of the volar surface of his left middle and ring finger with open fractures of the distal phalanx of the middle finger and avulsion of the digital nerves to the middle and ring fingers (-Fig. 1). He underwent immediate irrigation and open reduction, percutaneous pinning of the phalanx fracture, and placement of a vacuum closure device to temporarily cover the soft tissue received

May 19, 2018

accepted

June 18, 2018
DOI https://doi.org/

10.1055/s-0038-1676773. ISSN 2377-0813.
Copyright $\odot 2018$ by Thieme Medical Publishers, Inc., 333 Seventh Avenue, New York, NY 10001, USA. Tel: +1(212) 584-4662.
License terms

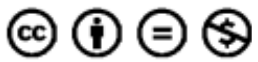




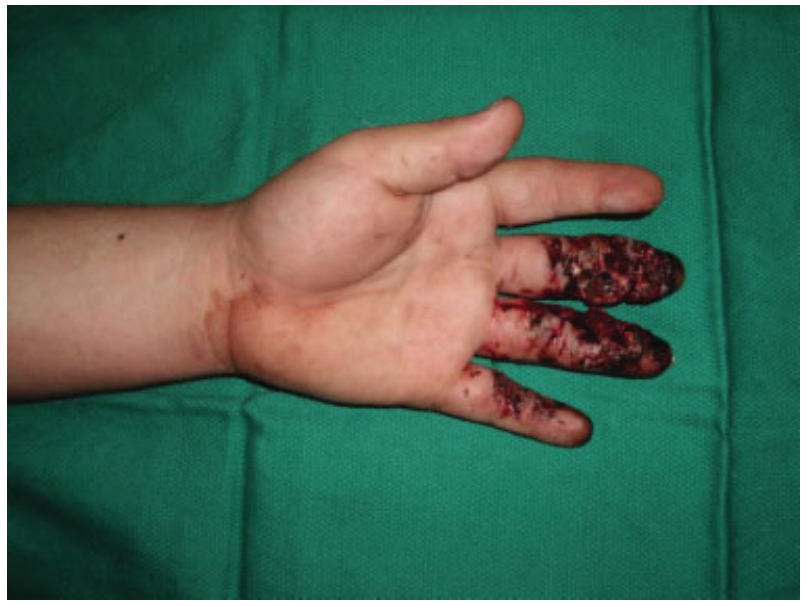

Fig. 1 Left hand multiple crush/avulsion injuries to volar middle and ring fingers distal to the proximal inter-phalangeal joint.

defect. The patient was taken back to the operating theater 2 days later for definitive closure of this large complex volar digital defect involving the index and middle finger. Given the unique location involving the volar surface of multiple digits, the decision to perform a free radial fascia only flap with split thickness skin graft and neosyndactylization of the affected digits was made. This would allow for a thin pliable flap over the distal fingers and improve the donor site defect.

An Allen test was performed preoperatively to verify that there was a patent arch with retrograde flow to the hand through the ulnar artery. The hand and arm were then exsanguinated, and the well-padded tourniquet was insufflated to $250 \mathrm{~mm} \mathrm{Hg}$. The volar wounds were then debrided, and the total wound on both fingers measured $6 \times 5 \mathrm{~cm}$. The neurovascular bundles were carefully identified and inspected. The ulnar digital nerve to the middle finger and the radial digital nerve to the ring finger were transected with a 2-cm segment loss at the level of the middle phalanx. These nerves were isolated and prepared for nerve grafting. An incision was extended proximally from the wound into the palm to explore and identify the common digital artery for use as our recipient vessel. A nearby dorsal vein was also isolated in the proximal web space and was prepared for our venous anastomosis.

Once the hand was prepared, we then turned our attention to harvesting the ipsilateral radial forearm fascia-only flap. The skin incision was designed in a straight line over the proximal volar forearm ending in a curvilinear fashion at the distal forearm (-Fig. 2A). Skin flaps were elevated radially and ulnarly, taking care to identify and preserve both the lateral antebrachial nerve and dorsal branches of the radial nerve in the subcutaneous tissue. Following elevation of skin and subcutaneous tissue, the radial forearm fascia was incised creating a $7 \mathrm{~cm} \times 6 \mathrm{~cm}$ flap to cover both volar defects to the middle and ring fingers ( - Fig. 2B). Perforators into the fascia from the radial artery were preserved. The radial artery and its venae comitantes were identified proximally and distally and were protected throughout the dissection. The fascial flap was elevated off the muscle bellies below, taking care to preserve the paratenon over the underlying tendons. Once the fascia and vessels were isolated to the intermuscular septum, the radial artery was clamped distally, and the tourniquet was released to confirm flow to the distal hand and fingers through the patent palmar arch. The distal artery was then ligated and divided. The flap was then elevated in a distal to proximal direction. The proximal pedicle of artery was designed to be roughly $8 \mathrm{~cm}$ in length. This was isolated and prepared for division.

Interposition nerve grafting of the ulnar digital nerve of the long finger and the radial digital nerve of the ring finger using a segment of the lateral antebrachial cutaneous nerve from the forearm was performed prior to insetting the flap. The pedicle of the radial forearm fascial flap was then divided, and the flap was partially inset using 4-0 chromic sutures to cover the exposed vital structures. The radial artery was anastomosed to the common digital artery in an end-to-side fashion using 8-0 nylon sutures in an interrupted fashion. After completion of the microvascular anastomosis all the digits were noted to be pink and well perfused with flow across the anastomosis. A primary hand sewn end-to-end microanastomosis was then performed from the venae comitantes of the radial artery to a dorsal vein in
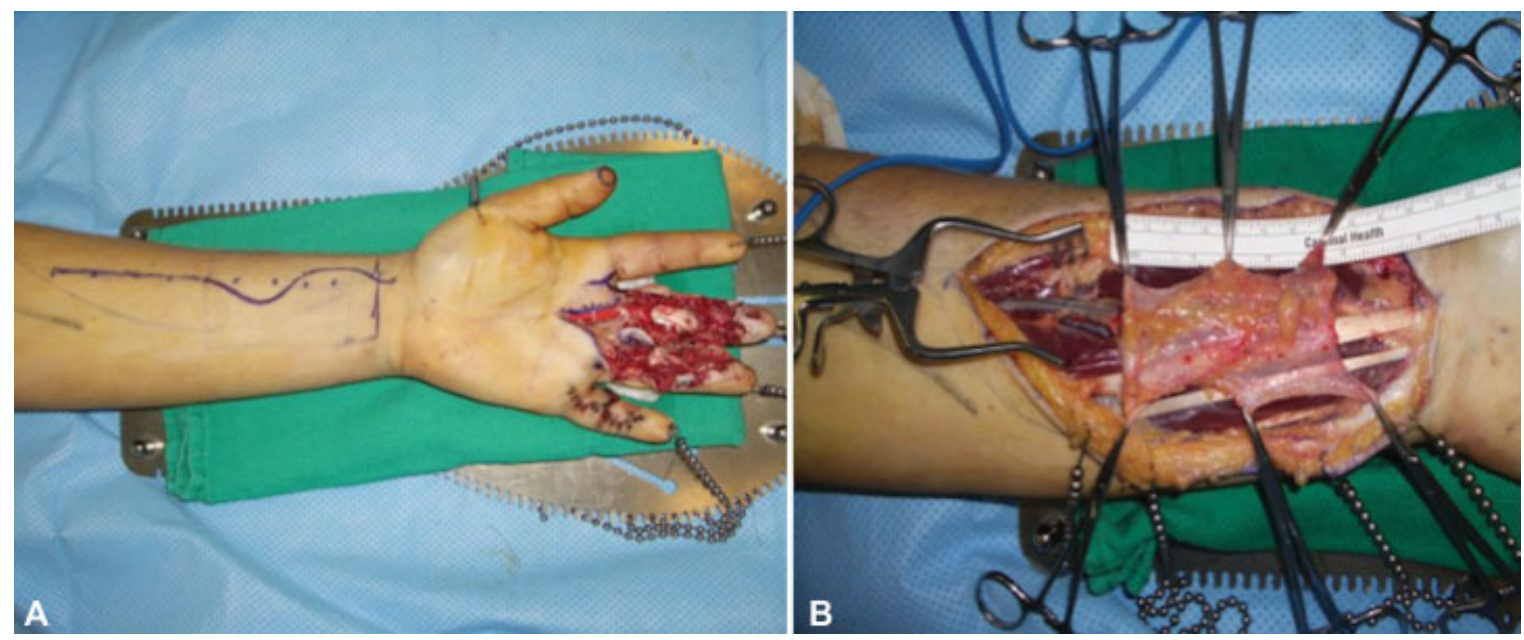

Fig. 2 Intraoperative dissection. (A) Design of volar skin incision. (B) Harvesting of the radial forearm fascia. 


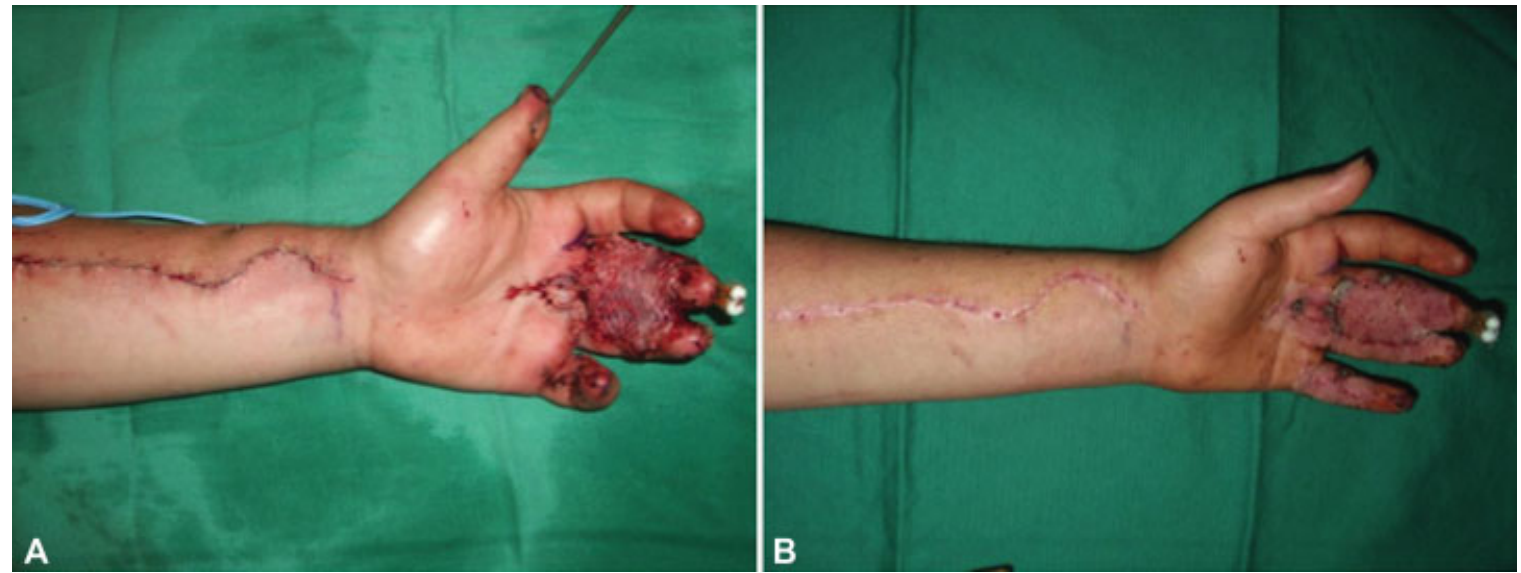

Fig. 3 (A) Completion of insetting and skin grafting with closure of donor site. (B) 3 months postoperative with well-healed donor site.

the third web space. An implantable venous Doppler probe was placed (Cook Vascular Inc, Vandergrift, PA) around the venous anastomosis. The fascial flap was then covered using a very thin split thickness skin graft harvested from the right thigh. Primary closure of the forearm donor site was easily performed (-Fig. 3). The patient was placed in a bulky dressing and a dorsal blocking splint to avoid tension across the nerve and vessels repairs. The flap survived without complications, and the patient underwent division of the neosyndactylized digits at 89 days from the original procedure without further complications or secondary procedures needed.

\section{Discussion}

Free flap transplantation allows for rapid closure of difficult wounds, provides a robust blood supply to the zone of injury, and facilitates clearing of infected wounds. Coverage of an upper extremity wound can be very challenging as it requires tissue that easily conforms to the contour of the forearm and hand, protects the underling structures, and provides a surface for tendon gliding. In our case, the volar surface of the left middle and ring fingers distal to the proximal interphalangeal joints were extensively damaged secondary to a crush/avulsion injury. Loco-regional flaps, such as cross finger flaps, were not possible due to defect size and injury to neighboring digits. ${ }^{1}$ Heterodigital or homodigital flaps may be of use in single injured digits with small defects. The reverse radial forearm flap is indicated for moderate-sized defects of the palmar and dorsal aspects of the wrist and hand out to the level of the proximal interphalangeal joints. ${ }^{6}$ However, our defects extended beyond this level, and any pedicled reverse radial forearm fasciocutaneous or adipofascial flaps would not likely be of adequate length. The free radial forearm flap has been used extensively for head and neck and upper extremity reconstruction. ${ }^{8}$ The disadvantage of this flap is significant donor site morbidity due to the inability to directly close the donor wound, thus requiring split thickness skin grafting. However, given the location and complexity of this injured region, we needed soft tissue coverage to be thin and pliable and allow for gliding of the underlying tendons. Ismail ${ }^{9}$ described a modification of this flap using only the forearm fascia as a free flap in eight cases. This modification not only allows for ease of donor site closure but also the use of a fasciaonly free flap to resurface the distal volar digits. Our case was further complicated due to the multiple injured digits requiring soft tissue coverage. When multiple adjacent fingers require soft tissue coverage, fascial flaps can be placed over the entire defect creating artificially syndactylized digits. This allows for complete coverage of all defects using a single free flap. This requires a second procedure to divide the flap after adequate neovascularization has occurred. Although many papers have been written on the subject, the exact timing at which free flaps become independent of their vascular pedicle remains unclear. Oswald et $\mathrm{al}^{10}$ showed that occlusion of the vascular pedicle in microvascular free flaps on the 5th day resulted in survival of the flap in rats. With the exception of anecdotal reports, most of the published literature suggests that free flaps in humans are dependent on their pedicle for at least 15 to 17 days. ${ }^{11-15}$ The condition and quality of the recipient site play a large role in survival of these flaps. Ischemic, irradiated, and scarred beds are inadequate in providing late flap neovascularization compared with healthy recipient sites and therefore would be dependent on the vascular pedicle for longer periods. ${ }^{16,17}$ In our patient, the decision and timing of flap division were altered by the patient's unavailability and lack of compliance.

In conclusion, the radial forearm free fascia (RFF) is a reliable flap with consistent vascular anatomy and low donor site morbidity. This thin and pliable flap allows for its use in locations where contour and gliding are critical. The fascia is also ample so one can modify the size of this flap to cover several neighboring defects with a single arterial anastomosis as was done in our case. Our case underscores the utility of the RFF free flap in the management of complex hand and digital injuries and its use as a single flap for multiple defects. We highly recommend its use and believe that it should be part of the armamentarium of the reconstructive surgeon.

Conflict of Interest

None declared. 
e90 Coverage of Two Separate Digital Defects Using a Single Fascial Free Flap with Neosyndactylization Herrera et al.

\section{References}

1 Curtis RM. Cross-finger pedicle flap in hand surgery. Ann Surg 1957;145(05):650-655

2 Rockwell WB, Lister GD. Soft tissue reconstruction. Coverage of hand injuries. Orthop Clin North Am 1993;24(03):411-424

3 Watumull D, Orenstein HH. Soft tissue coverage for the upper extremity. Orthopedics 1993;16(04):459-465

4 Page R, Chang J. Reconstruction of hand soft-tissue defects: alternatives to the radial forearm fasciocutaneous flap. J Hand Surg Am 2006;31(05):847-856

5 Chen HC, Buchman MT, Wei FC. Free flaps for soft tissue coverage in the hand and fingers. Hand Clin 1999;15(04):541-554

6 Jones NF, Jarrahy R, Kaufman MR. Pedicled and free radial forearm flaps for reconstruction of the elbow, wrist, and hand. Plast Reconstr Surg 2008;121(03):887-898

7 Strauch B, Yu HL. Atlas of Microvascular Surgery: Anatomy and Operative Approaches. New York: Thieme Medical Publishers; 1993

8 Soutar DS, Scheker LR, Tanner NS, McGregor IA. The radial forearm flap: a versatile method for intra-oral reconstruction. Br J Plast Surg 1983;36(01):1-8

9 Ismail TI. The free fascial forearm flap. Microsurgery 1989;10(03): $155-160$
10 Oswald P, Tilgner A, Schumann D. The influence of postoperative vessel occlusion on the viability of free microvascular skin-fat flaps and island flaps in rats. J Reconstr Microsurg 1988;4(05): 403-407

11 Gatti JE, LaRossa D, Brousseau DA, Silverman DG. Assessment of neovascularization and timing of flap division. Plast Reconstr Surg 1984;73(03):396-402

12 Chen HC, Tan BK, Cheng MH, Chang CH, Tang YB. Behavior of free jejunal flaps after early disruption of blood supply. Ann Thorac Surg 2002;73(03):987-989

13 Millican PG, Poole MD. Peripheral neovascularisation of muscle and musculocutaneous flaps. Br J Plast Surg 1985;38(03):369-374

14 Tsur H, Daniller A, Strauch B. Neovascularization of skin flaps: route and timing. Plast Reconstr Surg 1980;66(01):85-90

15 Clarke HM, Chen GZ. Peripheral neovascularization of muscle and musculocutaneous flaps in the pig. Plast Reconstr Surg 1992;89 (01):109-113, discussion 114-115

16 Morris SF, Yang D. Effect of vascular delay on viability, vasculature, and perfusion of muscle flaps in the rabbit. Plast Reconstr Surg 1999;104(04):1041-1047

17 Weinzweig N, Gonzalez M. Free tissue failure is not an all-or-none phenomenon. Plast Reconstr Surg 1995;96(03):648-660 